\title{
GEOMETRY OF CERTAIN LAGRANGIAN SUBMANIFOLDS IN HERMITIAN SYMMETRIC SPACES
}

\section{YOSHIHIRO OHNITA}

\begin{tabular}{|c|l|}
\hline Citation & OCAMI Preprint Series \\
\hline Issue Date & 2012 \\
\hline Type & Preprint \\
\hline Textversion & Author \\
\hline \multirow{2}{*}{ Rights } & $\begin{array}{l}\text { C } 2013 \text { World Scientific Publishing Company. For personal use only. No other } \\
\text { uses without permission. }\end{array}$ \\
\hline \multirow{2}{*}{ Relation } & $\begin{array}{l}\text { Preprint of an article submitted for consideration in Differential Geometry of } \\
\text { Submanifolds and its Related Topics } @ 2013 \text { World Scientific Publishing } \\
\text { Company. https://www.worldscientific.com/worldscibooks/10.1142/8970 . }\end{array}$ \\
\hline DOI & \begin{tabular}{l} 
It is published, it will be found at https://doi.org/10.1142/9789814566285_0006. \\
\hline
\end{tabular} \\
\hline
\end{tabular}

From: Osaka City University Advanced Mathematical Institute http://www.sci.osaka-cu.ac.jp/OCAMI/publication/preprint/preprint.html 


\title{
GEOMETRY OF CERTAIN LAGRANGIAN SUBMANIFOLDS IN HERMITIAN SYMMETRIC SPACES
}

\author{
YOSHIHIRO OHNITA
}

\begin{abstract}
In this paper we discuss a certain method to construct a family of Lagrangian submanifolds in a complex projective space $\mathbb{C} P^{n+1}$ from a Lagrangian submanifold in a complex hyperquadric $Q_{n}(\mathbb{C})$. We use a family of homogeneous isoparametric hypersurfaces in the unit standard sphere associated to the Riemannian symmetric pair $(S O(n+4), S O(2) \times S O(n+2))$ of rank 2 .
\end{abstract}

\section{INTRODUCTION}

It is an interesting problem to study the geometric construction of Lagrangian submanifolds in Hermitian symmetric spaces from the viewpoint of Lie theory. We shall give attention to a relationship among Lagrangian submanifolds in different Hermitian symmetric spaces of compact type.

In this paper we discuss a certain method to construct a family of Lagrangian submanifolds in a complex projective space $\mathbb{C} P^{n+1}$ from a Lagrangian submanifold in a complex hyperquadric $Q_{n}(\mathbb{C})$. We use a family of homogeneous isoparametric hypersurfaces in the unit standard sphere $S^{2 n+3}(1) \subset \mathbb{C}^{n+2}$ associated to the Riemannian symmetric pair $(S O(n+4), S O(2) \times S O(n+2))$ of rank 2. It induces a family of homogeneous isoparametric real hypersurfaces in a complex projective space $\mathbb{C} P^{n+1}$ whose focal manifolds are $Q_{n}(\mathbb{C})$ and $\mathbb{R} P^{n+1}$. Such a homogeneous real hypsersurface of a complex projective space is a tube over $Q_{n}(\mathbb{C})$ and $\mathbb{R} P^{n+1}$ and is called of type $B$ by $\mathrm{R}$. Takagi $([20,21])$. For each $t \in(-1,1)$, we have the positive focal map $\nu_{+}: M_{t} \rightarrow Q_{n}(\mathbb{C})$ and the negative focal map $\nu_{-}: M_{t} \rightarrow \mathbb{R} P^{n+1}$. By using the positive focal map $\nu_{+}: M_{t} \rightarrow Q_{n}(\mathbb{C})$, we shall show that for any Lagrangian submanifold $L$ in $Q_{n}(\mathbb{C})$ we have a family of Lagrangian submanifolds $\hat{L}^{n+1}=\nu_{+}^{-1}(L)\left(\subset M_{t}\right)$ in $\mathbb{C} P^{n+1}$ for $t \in(-1,1)$. We shall discuss their structure and properties in detail.

We shall also mention about known results on compact homogeneous Lagrangian submanifolds in complex projective spaces and the Gauss map construction of Lagrangian submanifolds in complex hyperquadrics $Q_{n}(\mathbb{C})$ from

Date: Dec. 31, 2012.

2010 Mathematics Subject Classification. Primary: 53C40; Secondary: 53D12, 53C42.

Key words and phrases: Lagrangian submanifold, complex projective space, Hamiltonian minimality, isoparametric hypersurface, real hypersurface.

This work was partially supported by JSPS Grant-in-Aid for Scientific Research (C) No. 24540090 . 
isoparametric hypersurfaces in $S^{n+1}(1)$. By applying our result to nice Lagrangian submanifolds in complex hyperquadrics, we can obtain many interesting examples of Lagrangian submanifolds in complex projective space. We expect that our result provides a link from Lagrangian submanifold theory in complex hyperquadrics to Lagrangian submanifold thery in complex projective spaces.

\section{LAGRANGIAN SUBMANIFOLDS IN KÄHLER MANIFOLDS}

A smooth immersion $\varphi: L \rightarrow P$ of a smooth manifold $L$ into a $2 n$ dimensional symplectic manifold $(P, \omega)$ is called a Lagrangian immersion if $\varphi^{*} \omega=0$ and $\operatorname{dim} L=n$. For each $v \in\left(\varphi^{-1} T P\right)_{x}=T_{\varphi(x)} P$, we define $\alpha_{v} \in T_{x}^{*} L$ by $\alpha_{v}(X):=\omega_{\varphi(x)}\left(v,(d \varphi)_{x}(X)\right)$ for each $X \in T_{x} L$. If $\varphi$ is a Lagrangian immersion, then we have the canonical isomorphisms $\varphi^{-1} T P / \varphi_{*} T L \cong T^{*} L$ and $C^{\infty}\left(\varphi^{-1} T P / \varphi_{*} T L\right) \cong \Omega^{1}(L)$. A smooth family of smooth immersions $\varphi_{t}: L \rightarrow$ $P$ with $\varphi_{0}=\varphi$ is called a Lagrangian deformation if $\varphi_{t}: L \rightarrow P$ is a Lagrangian immersion for each $t$. A Lagrangian deformation $\varphi_{t}: L \rightarrow P$ is characterized by the condition that $\alpha_{V_{t}}$ is closed for each $t$, where $V_{t}=\frac{\partial \varphi_{t}}{\partial t} \in C^{\infty}\left(\varphi_{t}^{-1} T P\right)$ is the variational vector field of $\left\{\varphi_{t}\right\}$. If $\alpha_{V_{t}}$ is exact for each $t$, then we call $\left\{\varphi_{t}\right\}$ a Hamiltonian deformation of $\varphi$.

Suppose that $(P, \omega, g, J)$ is a Kähler manifold with a Kähler metric $g$ and the complex structure $J$. Let $\varphi: L \rightarrow P$ be a Lagrangian immersion. Denote by $B$ the second fundamental form of $\varphi$. We define a symmetric tensor field $S$ on $L$ by

$$
S(X, Y, Z):=\omega(B(X, Y), Z)=g(J B(X, Y), Z)
$$

for each $X, Y, Z \in T L$. The mean curvature vector field $H$ of $\varphi$ is defined by

$$
H:=\sum_{i=1}^{n} B\left(e_{i}, e_{i}\right),
$$

where $\left\{e_{i}\right\}$ is an orthonormal basis of $T_{x} L$. The 1 -form $\alpha_{H}$ on $L$ corresponding to the mean curvature vector field $H$ is called the mean curvature form of $\varphi$, which satisfies the identity $([5])$

$$
d \alpha_{H}=\varphi^{*} \rho_{P}
$$

where $\rho_{P}$ denotes the Ricci form of a Käher manifold $P$.

The notion of Hamiltonian minimality for Lagrangian submanifolds in a Käher manifold $P$ was introduced and investigated first by Y. G. Oh ([12]). A Lagrangian immersion $\varphi: L \rightarrow P$ into a Käher manifold $P$ is called Hamiltonian minimal if for each compactly supported Hamiltonian deformation $\left\{\varphi_{t}\right\}$ of $\varphi_{0}=\varphi$ the first variation of the volume vanishes:

$$
\left.\frac{d}{d t} \operatorname{Vol}\left(L, \varphi^{*} g\right)\right|_{t=0}=0 .
$$

The Hamiltonian minimal Lagrangian submanifold equation is

$$
\delta \alpha_{H}=0 .
$$


The usual minimal submanifold equation $H=0$ is equivalent to $\delta \alpha_{H}=0$ and in this case we call $\varphi$ a minimal Lagrangian immersion. A Hamiltonian Lagrangian immersion $\varphi: L \rightarrow P$ into a Käher manifold $P$ is called Hamiltonian stable if for each compactly supported Hamiltonian deformation $\left\{\varphi_{t}\right\}$ of $\varphi_{0}=\varphi$ the second variation of the volume is nonnegative:

$$
\left.\frac{d^{2}}{d t^{2}} \operatorname{Vol}\left(L, \varphi^{*} g\right)\right|_{t=0} \geq 0
$$

The Lagrangian version of the second variational formula was given in [12].

By considering the second variation of the volume for Hamiltonian minimal Lagrangian submanifolds under Hamiltonian deformations, the notions of Hamiltonian stability and strict Hamiltonian stability are defined (cf. [12], [8]).

Let $G$ be a connected Lie group acting on $P$ preserving symplectic form $\omega$. A Lagrangian submanifold $L$ embedded in a symplectic manifold $(P, \omega)$ obtained as an orbit of $G$ is called a Lagrangian orbit of $G$.

Assume that $(P, \omega, J, g)$ is a Kähler manifold and the action of $G$ on $P$ preserves the Kähler structure $(\omega, g, J)$, or $G$ is a connected Lie subgroup of the automorphism group $\operatorname{Aut}(P, \omega, J, g)$ of the Kähler structure $(\omega, g, J)$. If a Lagrangian submanifold $L$ embedded in $P$ is obtained as an orbit of $G$, then $L$ is called a homogeneous Lagrangian submanifold in a Kähler manifold $P$. Moreover if $L$ is compact without boundary, then $L$ is said to be a compact homogeneous Lagrangian submanifold in a Kähler manifold $P$. In this case, as $\delta \alpha_{H}$ is a constant function on $L$, by the divergence theorem we have $\delta \alpha_{H}=0$. Hence we know

Proposition 1.1 ([8]). Any compact homogeneous Lagrangian submanifold in a Käher manifold is always Hamiltonian minimal.

Suppose that $(P, \omega, J, g)$ is a compact Kähler manifold with $\operatorname{dim}_{\mathbb{C}} H^{1,1}(P, \mathbb{C})=$ 1 and $G$ is a compact connected Lie subgroup of the automorphism group $\operatorname{Aut}(P, \omega, J, g)$. Let $G^{\mathbb{C}} \subset \operatorname{Aut}(P, J)$ be the complexified Lie group of $G$. Then the following is a fundamental fact on the existence of compact homogeneous Lagrangian submanifolds in Kähler manifolds.

Theorem ([4]). If $G \cdot x$ is a Lagrangian orbit of $G$, then the complexified orbit $G^{\mathbb{C}} \cdot x$ is Stein and Zariski open in $P$. Conversely if the complexified orbit $G^{\mathbb{C}} \cdot x$ is Stein and Zariski open in $P$, then $G^{\mathbb{C}} \cdot x$ contains a Lagrangian orbit of $G$.

\section{LAGRANGIAN SUBMANIFOLDS IN COMPLEX PROJECTIVE SPACES}

Let $\mathbb{C}^{n+1}$ be the complex Euclidean space and $S^{2 n+1}(1)$ be the unit standard hypersphere of $\mathbb{C}^{n+1} \cong \mathbb{R}^{2 n+2}$. Let $\pi: S^{2 n+1}(1) \rightarrow \mathbb{C} P^{n}$ be the standard Hopf fibration over the $n$-dimensional complex projective space $\mathbb{C} P^{n}$ equipped with the standard Fubini-Study metric of constant holomorphic sectional curvature 4. Note that the standard $S^{1}$-action on $S^{2 n+1}(1)$ preserves the induced metrics. 
and $\pi: S^{2 n+1}(1) \rightarrow \mathbb{C} P^{n}$ is a Riemannian submersion. The energy function $f: \mathbb{C}^{n+1} \rightarrow \mathbb{R}$ defined by $f(\mathbf{x}):=\|\mathbf{x}\|^{2}\left(\mathbf{x} \in \mathbb{C}^{n+1}\right)$ can be considered as a Hamiltonian function or a moment map with respect to the standard $S^{1}$-action on $\mathbb{C}^{n+1}$. Since $f^{1}=S^{2 n+1}(1)$ and $f^{1} / S^{1}=\mathbb{C} P^{n}$, the complex projective space $\mathbb{C} P^{n}$ is a symplectic Kähler quotient of $\mathbb{C}^{n+1}$ by the moment map $f$. Then we know the following properties (cf. [6]):

Proposition 2.1. Let $L$ be a submanifold (immersed) in $\mathbb{C} P^{n}$. Then

(1) $L$ is a Lagrangian submanifold (immersed) in $\mathbb{C} P^{n}$ if and only if the inverse image $\tilde{L}=\pi^{-1}(L)\left(\subset S^{2 n+1}(1)\right)$ by the Hopf fibration $\pi$ is a Lagrangian submanifold (immersed) in $\mathbb{C}^{n+1}$.

(2) $L$ is a Hamiltonian minimal in $\mathbb{C} P^{n}$ if and only if $\tilde{L}=\pi^{-1}(L)(\subset$ $\left.S^{2 n+1}(1)\right)$ is Hamiltonian minimal in $\mathbb{C}^{n+1}$.

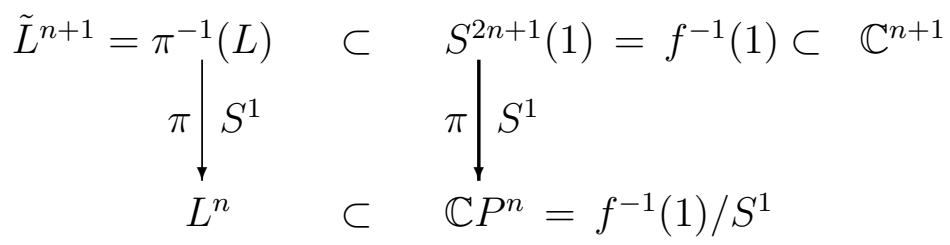

We mention about known results on the construction and classification of compact homogeneous Lagrangian submanifolds in complex projective spaces. In the case when $L$ has parallel second fundamental form, that is, $\nabla S=$ $0, \pi^{-1}(L)$ also has parallel second fundamental form in both $S^{2 n+1}(1)$ and $\mathbb{C}^{n+1}$. Such Lagrangian submanifolds with $\nabla S=0$ in complex space forms were completely classified by Professors Hiroo Naitoh and Mararu Takeuchi in 1980's by theory of symmetric R-spaces (of type $U(r)$ ) and in particular they showed that any Lagrangian submanifolds with $\nabla S=0$ in a complex space form is homogeneous. Compact Lagrangian submanifolds with $\nabla S=0$ embedded in complex projective spaces and complex Euclidean spaces are strictly Hamiltonian stable (Amarzaya-Ohnita [1], [2]).

There are many examples of compact homogeneous Lagrangian submanifolds with $\nabla S \neq 0$ embedded in complex projective spaces and complex Euclidean spaces. The simplest non-trivial example can be given by a 3dimensional minimal Lagrangian orbit in $\mathbb{C} P^{2}$ under an irreducible unitary representation of $S U(2)$ of degree 3 (cf. [3], [13]).

Bedulli-Gori ([4]) classified compact homogeneous Lagrangian submanifolds in complex projective spaces obtained as orbits of simple compact Lie groups of $P U(n+1)$. It is a crucial to use Theorem and theory of prehomogeneous vector spaces due to Professors Mikio Sato and Tatsuo Kimura. It is still an open problem to classify compact homogeneous Lagrangian submanifolds in $\mathbb{C} P^{n}$ obtained as orbits of non-simple compact Lie groups of $P U(n+1)$ (See also [19]). 


\section{LAGRANGIAN SUBMANIFOLDS IN COMPLEX HYPERQUADRICS AND HYPERSURFACE GEOMETRY IN SPHERES}

Let $N^{n}$ be an oriented hypersurface immersed in the unit standard sphere $S^{n+1}(1)$. Let $\mathbf{x}(p)$ denote the position vector of a point $p \in N^{n}$ and $\mathbf{n}(p)$ denote the unit normal vector at $p \in N^{n}$ in $S^{n+1}(1)$. The Gauss map $\mathcal{G}: N^{n} \rightarrow Q_{n}(\mathbb{C})$ of the oriented hypersurface $N^{n} \subset S^{n+1}(1)$ is defined by

$$
\mathcal{G}: N^{n} \ni p \longmapsto[\mathbf{x}(p)+\sqrt{-1} \mathbf{n}(p)]=\mathbf{x}(p) \wedge \mathbf{n}(p) \in Q_{n}(\mathbb{C})=\widetilde{G r_{2}}\left(\mathbb{R}^{n+2}\right)
$$

It is well-known that the Gauss map $\mathcal{G}: N^{n} \rightarrow Q_{n}(\mathbb{C})$ is always a Lagrangian immersion.

We shall assume that $N^{n}$ is an oriented isoparametric hypersurface immersed in the unit standard sphere $S^{n+1}(1)$, that is, a hypersurface with $g$ distinct constant principal curvatures.

In general, an isoparametric hypersurface on a Riemannian manifold $M$ is defined as a regular level hypersurface of an isoparametric function. A smooth function $f$ is called an isoparametric function on a Riemannian manifold $M$ if $f$ satisfies the system of partial differential equations:

$$
\Delta^{M} f=a(f), \quad\|\operatorname{grad} f\|^{2}=b(f),
$$

where $a$ is a continuous function and $b$ is a function of class $C^{2}$. It is wellknown that each regular level hypersurface of an isoparametric function has constant mean curvature. Elie Cartan showed that a hypersurface immersed in real space forms is an isoparametric hypersurface if and only if it has constant principal curvatures. Let $N^{n}$ be an isoparametric hypersurface in the standard hypersphere $S^{n+1}(1) \subset \mathbb{R}^{n+2}$ with $g$ distinct principal curvatures $k_{1}>k_{2}>$ $\cdots>k_{g}$ and the corresponding multiplicities $m_{\alpha}(\alpha=1, \cdots, g)$. We express the principal curvatures $k_{1}>k_{2}>\cdots>k_{g}$ as $k_{\alpha}=\cot \theta_{\alpha}(\alpha=1, \cdots, g)$ with $\theta_{1}<\cdots<\theta_{g}$. Münzner $([10])$ showed that $\theta_{\alpha}=\theta_{1}+(\alpha-1) \frac{\pi}{g}$ and $m_{\alpha}=m_{\alpha+2}$ indexed modulo $g$. Moreover he proved that $N^{n}$ extends to a regular level hypersurface $f^{-1}(t)$ for some $t \in(-1,1)$ defined by an isoparametric function $f: S^{n+1}(1) \rightarrow[-1,1]$ which is a restriction of a homogeneous polynomial $F$ of degree $g$ satisfying

$$
\Delta^{\mathbb{R}^{n+2}} F=c r^{g-2}, \quad\left\|\operatorname{grad}^{\mathbb{R}^{n+2}} F\right\|^{2}=g^{2} r^{2 g-2},
$$

where $r=\sum_{i=1}^{n+2}\left(x_{i}\right)^{2}$ and $c=g^{2}\left(m_{2}-m_{1}\right) / 2$. Here the isoparametric function $f$ is defined in a neighborhood of $N^{n}$ as

$$
t=f(q)=\cos (g \theta(q))=\cos \left(g \theta_{1}\right),
$$

where $\theta(q):=\theta_{1}$ for the maximal principal curvature $k_{1}=\cot \theta_{1}$ of $f^{-1}(q)$. Such a homogeneous polynomial is called the Cartan-Münzner polynomial. His famous result is that $g$ must be $1,2,3,4$ or 6 ([11]) .

Let $k_{1}>\cdots>k_{g}$ denote its distinct constant principal curvatures and $m_{1}, \cdots, m_{g}$ the corresponding multiplicities. Münzner $([10])$ showed that $\theta_{\alpha}=$ 
$\theta_{1}+(\alpha-1) \frac{\pi}{g}$ and $m_{\alpha}=m_{\alpha+2}$ indexed modulo $g$. Hence we have

$$
\frac{2 n}{g}= \begin{cases}m_{1}+m_{2} & \text { if } g \geq 2 \\ 2 m_{1} & \text { if } g=1\end{cases}
$$

The famous result of Münzner is that $g$ must be $1,2,3,4$ or 6 ([10], [11]).

The Lagrangian immersions $\mathcal{G}$ and the Gauss image $\mathcal{G}\left(N^{n}\right)$ have the following properties. It follows from the mean curvature form formula of Palmer ([18]) that

Proposition 3.1 ([18]). The Gauss map $\mathcal{G}: N^{n} \rightarrow Q_{n}(\mathbb{C})$ is a minimal Lagrangian immersion.

Proposition 3.2 ([7], [14]). The Gauss image $\mathcal{G}\left(N^{n}\right)$ is a compact smooth minimal Lagrangian submanifold embedded in $Q_{n}(\mathbb{C})$ and the Gauss map $\mathcal{G}$ : $N^{n} \rightarrow \mathcal{G}\left(N^{n}\right)$ is a covering map with the deck transformation group $\mathbb{Z}_{g}$. Moreover, $\mathcal{G}\left(N^{n}\right)$ is a monotone and cyclic Lagrangian submanifold in $Q_{n}(\mathbb{C})$ with minimal Maslov number $\frac{2 n}{g}$. Here $\frac{2 n}{g}$ is even if and only if $\mathcal{G}\left(N^{n}\right)$ is orientable.

In [7] we classified explicitly all compact homogeneous Lagrangian submanifolds in $Q_{n}(\mathbb{C})$. In particular we showed that all compact homogeneous minimal Lagrangian submanifolds in $Q_{n}(\mathbb{C})$ are obtained as the Guass images of homogeneous isoparametric hypersurfaces in $S^{n+1}(1)$.

Palmer $([18])$ showed that $\mathcal{G}: N^{n} \rightarrow Q_{n}(\mathbb{C})$ is Hamiltonian stable if and only if $g=1$, that is, $N^{n}$ is a great or small sphere of $S^{n+1}(1)$. By my joint works [7], [9] with Hui Ma, we completely determined the strict Hamiltonian stability of the Gauss images $\mathcal{G}\left(N^{n}\right)$ of all homogeneous isoparametric hypersurfaces $N^{n}$ in $S^{n+1}(1)$. Our main result was that the Gauss image $\mathcal{G}\left(N^{n}\right)$ of homogeneous isoparametric hypersurfaces $N^{n}$ is Hamiltonian stable if and only if $\left|m_{1}-m_{2}\right| \leq 2$ or $N^{n}$ is a principal orbit of the isotropy representation of the Riemannian symmetric pair of type EIII (in this case $\left(m_{1}, m_{2}\right)=(6,9)$ ).

\section{Construction of a FAMily of Lagrangian SUBMANifolds in $\mathbb{C} P^{n+1}$ From AN ARBITRARY LAGRANGIAN SUBMANIFOLD IN $Q_{n}(\mathbb{C})$}

First we recall the structure of tubes $M_{t}(t \in(-1,1))$ over a complex hyperquadric $Q_{n}(\mathbb{C})\left(=M_{1}=M_{+}\right)$in $\mathbb{C} P^{n+1}$. They are also tubes $M_{t}(t \in(-1,1))$ over a real projective subspace $\mathbb{R} P^{n}\left(=M_{-1}=M_{-}\right)$in $\mathbb{C} P^{n+1}$ at the same time. The tube $M_{t}$ is a real hypersurface embedded in $\mathbb{C} P^{n+1}$, which is a homogeneous isoparametric real hypersurface of $\mathbb{C} P^{n+1}$ and its focal manifolds are $Q_{n}(\mathbb{C})$ and $\mathbb{R} P^{n}$. Let $\nu$ denote the unit normal vector field to $M_{t}$ in $\mathbb{C} P^{n+1}$. The focal maps from $M_{t}$ to focal manifolds are defined by using geodesics of $\mathbb{C} P^{n+1}$ normal to $M_{t}$. Let $\nu_{+}: M_{t} \rightarrow Q_{n}(\mathbb{C})$ denote the positive focal map from $M_{t}$ onto $Q_{n}(\mathbb{C})$ and $\nu_{-}: M_{t} \rightarrow \mathbb{R} P^{n}$ the negative focal map from $M_{t}$ onto $\mathbb{R} P^{n}$ : 


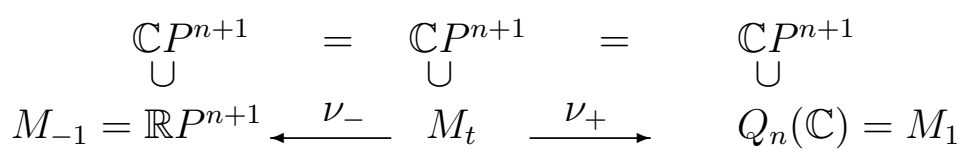

The homogeneous isoparametric real hypersurfaces $M_{t}$ in $\mathbb{C} P^{n+1}$ are obtained as the projection of homogeneous isoparametric real hypersurfaces $\tilde{M}_{t}$ in $S^{2 n+3}(1)$ by the Hopf fibration $\pi: S^{2 n+3}(1) \rightarrow \mathbb{C} P^{n+1}$. Let $(\tilde{G}, \tilde{U})=(S O(n+4), S O(2) \times$ $S O(n+2))$ be a compact rank 2 Riemannian symmetric pair of type $\mathrm{BDII}_{2}$. Let $\tilde{\mathfrak{g}}=\tilde{\mathfrak{u}}+\tilde{\mathfrak{p}}$ be the corresponding canonical decomposition of its symmetric Lie algebra, where $\tilde{\mathfrak{g}}=\mathfrak{o}(n+4), \tilde{\mathfrak{u}}=\mathfrak{o}(2) \oplus \mathfrak{o}(n+2)$ and the vector subspace $\tilde{\mathfrak{p}}$ is

$$
\tilde{\mathfrak{p}}=\left\{\left[\begin{array}{cc}
0 & \xi \\
-\xi^{t} & 0
\end{array}\right] \mid \xi \in M(2, n+2 ; \mathbb{R})\right\} .
$$

Here $M(2, n+2 ; \mathbb{R})$ denotes the vector space of all real $2 \times(n+2)$-matrices. The standard complex structure $\tilde{J}$ of $\tilde{\mathfrak{p}}$ invariant under the isotropy representation of $\tilde{U}$ is given by

$$
\begin{aligned}
J\left(\left[\begin{array}{cc}
0 & \xi \\
-\xi^{t} & 0
\end{array}\right]\right) & =\operatorname{Ad}_{\tilde{p}}\left[\begin{array}{ccc}
0 & -1 & 0 \\
1 & 0 & 0 \\
0 & 0 & 0
\end{array}\right]\left(\left[\begin{array}{cc}
0 & \xi \\
-\xi^{t} & 0
\end{array}\right]\right) \\
& =\left[\begin{array}{cc}
0 & {\left[\begin{array}{cc}
0 & -1 \\
1 & 0
\end{array}\right] \xi} \\
-\xi^{t}\left[\begin{array}{cc}
0 & -1 \\
1 & 0
\end{array}\right]^{t} & 0
\end{array}\right] .
\end{aligned}
$$

Relative to the standard complex structure, the vector subspace $\tilde{\mathfrak{p}}$ is identified with

$$
M(2, n+2 ; \mathbb{R}) \cong \mathbb{R}^{2 n+4} \cong \mathbb{C}^{n+2} .
$$

In particular, the standard $S^{1}$-action on $\mathbb{C}^{n+2}$ coincides with the isotropy action of $S O(2) \times\left\{\mathrm{I}_{n+2}\right\} \subset S O(2) \times S O(n+2)$ on $\tilde{\mathfrak{p}}$.

Let $S^{2 n+3}(1) \subset \tilde{\mathfrak{p}}$ denote the unit standard hypersphere of $\tilde{\mathfrak{p}}$. Each principal orbit of $S O(2) \times S O(n+2)$ on $S^{2 n+3}(1)$ is of codimension 1 . Thus we have a family $\left\{\tilde{M}_{t}\right\}$ of homogeneous hypersurfaces of $S^{2 n+3}(1)$ with constant principal curvatures, which is a family of so-called isoparametric hypersurfaces. Each $M_{t}$ has $g=4$ distinct principal curvatures and multiplicities $\left(m_{1}, m_{2}\right)=(1, n)$.

Let $\tilde{\tilde{f}}: S^{2 n+3}(1) \rightarrow[-1,1] \subset \mathbb{R}$ be such an isoparametric function defining $\left\{\tilde{M}_{t}\right\}$ and $F: \mathbf{C}^{n+2} \rightarrow \mathbb{R}$ denote the corresponding Cartan-Münzner polynomial of degree 4 so that $\left.F\right|_{S^{2 n+3}(1)}=\tilde{f}$. Explicitly the Cartan-Münzner polynomial $F$ is given as follows ([17]):

$$
F(Z)=\frac{3}{4}\left(\operatorname{Tr}\left(Z^{2}\right)\right)^{2}-2 \operatorname{Tr}\left(Z^{4}\right)
$$

for each $Z \in \tilde{\mathfrak{p}}$, in other words

$$
F=r^{4}-2 F_{0},
$$


where the functions $r$ and $F$ are defined as

$$
r(\xi):=\left(\sum_{i=1}^{n+2}\left|\xi_{i}\right|^{2}\right)^{\frac{1}{2}}, \quad F_{0}(\xi):=\left|\sum_{i=1}^{n+2} \xi_{i}^{2}\right|^{2}=\left(\sum_{i=1}^{n+2} \xi_{i}^{2}\right) \overline{\left(\sum_{i=1}^{n+2} \xi_{i}^{2}\right)}
$$

for each $\xi=\left[\begin{array}{c}\xi_{1} \\ \vdots \\ \xi_{n+2}\end{array}\right] \in \mathbb{C}^{n+2}$.

Set $\tilde{M}_{t}:=\tilde{f}^{-1}(t)$ for each $t \in[-1,1]$ and $\tilde{M}_{ \pm}:=\tilde{M}_{ \pm 1}$. Then for each $t \in$ $(-1,1)$ the level regular hypersurface $\tilde{M}_{t}$ is a compact homogeneous isoparametric hypersurface embedded in $S^{2 n+3}(1)$ which is diffeomorphic to a compact homogeneous space $\frac{S O(2) \times S O(n+2)}{\mathbb{Z}_{2} \times S O(n)}$. For $t= \pm 1, \tilde{M}_{ \pm}$are focal manifolds which are compact minimal submanifolds embedded in $S^{2 n+3}(1)$. A focal manifold $\tilde{M}_{+}$is diffeomorphic to a compact homogeneous space $\frac{S O(2) \times S O(n+2)}{S O(2) \times S O(n)}$ and another focal manifold $\tilde{M}_{-}$is diffeomorphic to a compact homogeneous space $\frac{S O(2) \times S O(n+2)}{O(n+1)}\left(\cong S^{1} \cdot S^{n+1} \cong Q_{2, n+2}(\mathbb{R})\right.$ a real hyperquadric $)$.

Let $t \in(-1,1)$ and set $\tilde{M}=\tilde{M}_{t}$. Let $k_{1}>k_{2}>k_{3}>k_{4}$ be four distinct principal curvatures of $M$ in $S^{2 n+3}(1)$. Recall that $k_{1}=\cot \theta_{1}$ and $t=\cos \left(4 \theta_{1}\right)$. Denote by $\mathbf{x}$ the position vector of points on $\tilde{M}$ and by $\mathbf{n}:=\frac{\operatorname{grad} \tilde{f}}{\|\operatorname{grad} \tilde{f}\|}$ the unit normal vector to $\tilde{M}$ in $S^{2 n+3}(1)$. Let $A_{\mathbf{n}}$ denotes the shape operator of $\tilde{M}$ in $S^{2 n+3}(1)$ in the direction of $\mathbf{n}$. The principal curvatures are nothing but eigenvalues of $A_{\mathbf{n}}$. For each $p \in \tilde{M}$, we express the eigenspace decomposition of the tangent vector space $T_{p} \tilde{M}$ corresponding to the four constant principal curvatures as follows:

$$
\begin{aligned}
T_{p} \tilde{M} & =\tilde{V}_{1}(p) \oplus \tilde{V}_{2}(p) \oplus \tilde{V}_{3}(p) \oplus \tilde{V}_{4}(p) \\
& =\mathbb{R} J \mathbf{x}(p) \oplus \mathbb{R} J \mathbf{n}(p) \oplus \tilde{V}_{2}(p) \oplus J\left(\tilde{V}_{2}(p)\right),
\end{aligned}
$$

where $\tilde{V}_{1}(p)=\mathbb{R}\left(-k_{3} J \mathbf{x}(p)+J \mathbf{n}(p)\right), \tilde{V}_{3}=\mathbb{R}\left(-k_{1} J \mathbf{x}(p)+J \mathbf{n}(p)\right), \tilde{V}_{4}(p)=$ $J\left(\tilde{V}_{2}(p)\right)$.

The positive focal map $\tilde{\nu}_{+}: \tilde{M}_{t} \rightarrow \tilde{M}_{+}$is defined by

$$
\tilde{\nu}_{+}(p):=\cos \theta_{1} \mathbf{x}(p)+\sin \theta_{1} \mathbf{n}(p) \in \tilde{M}_{+}
$$

for each $p \in \tilde{M}_{t}$. The differential of the positive focal map $\tilde{\nu}_{+}$is given as

$$
\begin{aligned}
&\left(d \tilde{\nu}_{+}\right)_{p}(X)=\cos \theta_{1} X-\sin \theta_{1} A_{\mathbf{n}}(X) \\
&=\left(\cos \theta_{1} I-\sin \theta_{1} A_{\mathbf{n}}\right)(X) \\
& 8
\end{aligned}
$$


for each $X \in T_{p} \tilde{M}$. If $X \in \tilde{V}_{i}(p)(i=1,2,3,4)$, then we have

$$
\begin{aligned}
\left(d \tilde{\nu}_{+}\right)_{p}(X) & =\left(\cos \theta_{1}-\sin \theta_{1} k_{i}\right)(X) \\
& =\left(\cos \theta_{1}-\sin \theta_{1} \cot \theta_{i}\right)(X) \\
& =\frac{\sin \left(\theta_{i}-\theta_{1}\right)}{\sin \theta_{i}} X .
\end{aligned}
$$

Note that

$$
\frac{\sin \left(\theta_{i}-\theta_{1}\right)}{\sin \theta_{i}}= \begin{cases}0 & \text { if } \theta_{i}=\theta_{1} . \\ \frac{1}{\sqrt{2} \sin \theta_{2}}=\frac{1}{\cos \theta_{1}+\sin \theta_{1}} & \text { if } \theta_{i}=\theta_{2} . \\ \frac{1}{\sin \theta_{3}}=\frac{1}{\cos \theta_{1}} & \text { if } \theta_{i}=\theta_{3} . \\ \frac{1}{\sqrt{2} \sin \theta_{4}}=\frac{1}{\sqrt{2} \cos \theta_{2}}=\frac{1}{\cos \theta_{1}-\sin \theta_{1}} & \text { if } \theta_{i}=\theta_{4}\end{cases}
$$

The positive focal map $\tilde{\nu}_{+}: \tilde{M}_{t} \rightarrow \tilde{M}_{+}$is an $S O(2) \times S O(n+2)$-equivariant submersion

$$
\begin{aligned}
\nu_{+}: \tilde{M}_{t}=\frac{S O(2) \times S O(n+2)}{\mathbb{Z}_{2} \times S O(n)} \longrightarrow \tilde{M}_{+} & =\frac{S O(2) \times S O(n+2)}{S O(2) \times S O(n)} \\
& \cong \frac{S O(n+2)}{S O(n)} \cong V_{2}\left(\mathbb{R}^{n+2}\right)
\end{aligned}
$$

with fiber $\frac{S O(2) \times S O(n)}{\mathbb{Z}_{2} \times S O(n)} \cong \frac{S O(2)}{\mathbb{Z}_{2}} \cong S^{1}$. Here $V_{2}\left(\mathbb{R}^{n+2}\right)$ denotes the Stiefel manifold of all pairs of two orthonormal vectors in $\mathbb{R}^{n+2}$. Note that this map $\tilde{\nu}_{+}: \tilde{M}_{t} \rightarrow \tilde{M}_{+}$is not a Riemannian submersion.

Let $\pi: S^{2 n+3}(1) \rightarrow \mathbb{C} P^{n+1}$ be the Hopf fibration with the $S^{1} \cong S O(2)$ action. Since the isoparametric function $\tilde{f}$ on $S^{2 n+3}(1)$ is invariant under the $S^{1} \cong S O(2)$-action, a smooth function $f: \mathbb{C} P^{n+1} \rightarrow[-1,1] \subset \mathbb{R}$ can be induced by $f \circ \pi=\tilde{f}$. Then $f$ is an isoparametric function on $\mathbb{C} P^{n+1}$.

Set $M_{t}:=f^{-1}(t)$ for each $t \in[-1,1]$ and $M_{ \pm}:=M_{ \pm 1}$. Then for each $t \in(-1,1)$ the subset $M_{t}$ is the level regular hypersurface, and is a compact homogeneous isoparametric hypersurface embedded in $\mathbb{C} P^{n+1}$ which is diffeomorphic to $\frac{S O(n+2)}{\mathbb{Z}_{2} \times S O(n)}$. The subsets $M_{ \pm}$are focal manifolds which are compact minimal submanifolds embedded in $\mathbb{C} P^{n+1}$. The subset $M_{+}$is a complex hyperquadric $Q_{n}(\mathbb{C})$ in $\mathbb{C} P^{n+1}$ which is diffeomorphic to $\frac{S O(n+2)}{S O(2) \times S O(n)}$ and the subset $M_{-}$is a real projective subspace $\mathbb{R} P^{n+1}$ in $\mathbb{C} P^{n+1}$ which is diffeomorphic to $\frac{S O(n+2)}{S O(1) \times S O(n+1)}$.

Each $M_{t}(t \in(-1,1))$ is a homogeneous isoparametric real hypersurface in $\mathbb{C} P^{n+1}$ with three distinct constant principal curvatures

$$
\ell_{1}=-2 \tan 2 \theta_{1}, \ell_{2}=\cot \theta_{1}, \ell_{3}=-\tan \theta_{1},
$$


and the corresponding multiplicities

$$
m\left(\ell_{1}\right)=1, m\left(\ell_{2}\right)=n, m\left(\ell_{3}\right)=n .
$$

It is called a homogeneous real hypersurface of type $B([20,21])$. The vector field $\nu:=\frac{\operatorname{grad} f}{\|\operatorname{grad} f\|}$ is a unit normal vector field on each $M_{t}(t \in(-1,1))$ and $A_{\nu}$ denotes the shape operator of $M_{t}$ in the direction of $\nu$. For each $x \in M_{t}$, we express the eigenspace decomposition of $T_{x} M_{t}$ corresponding to three principal curvatures $\ell_{1}, \ell_{2}, \ell_{3}$ as follows:

$$
T_{x} M_{t}=V_{1}(x) \oplus V_{2}(x) \oplus V_{3}(x)
$$

where $V_{1}(x)=\mathbb{R} J \nu(x), V_{2}(x)=J\left(V_{3}(x)\right)$.

Now we have a commutative diagram of positive focal maps and Hopf fibrations as follows:

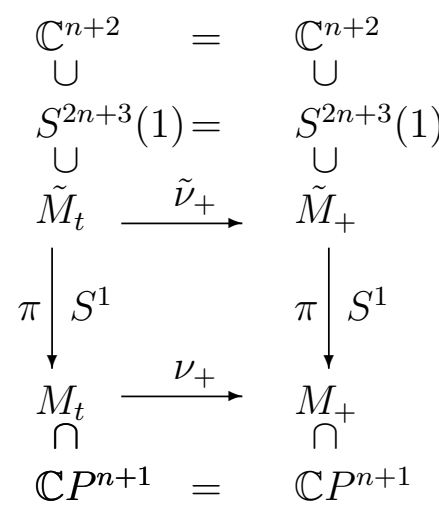

Here

$$
\begin{aligned}
& \tilde{M}_{t}=\pi^{-1}\left(M_{t}\right) \cong \frac{S O(2) \times S O(n+2)}{\mathbb{Z}_{2} \times S O(n)} \quad(t \in(-1,1)), \\
& \tilde{M}_{+}=\pi^{-1}\left(M_{+}\right) \cong \frac{S O(2) \times S O(n+2)}{S O(2) \times S O(n)} \cong \frac{S O(n+2)}{S O(n)} \cong V_{2}\left(\mathbb{R}^{n+2}\right), \\
& \tilde{M}_{-}=\pi^{-1}\left(M_{-}\right) \cong \frac{S O(2) \times S O(n+2)}{O(n+1)} \cong S^{1} \cdot S^{n+1} \cong Q_{2, n+2}(\mathbb{R}),
\end{aligned}
$$

and

$$
\begin{aligned}
& M_{t}=\pi\left(\tilde{M}_{t}\right) \cong \frac{S O(n+2)}{\mathbb{Z}_{2} \times S O(n)} \quad(t \in(-1,1)), \\
& M_{+}=\pi\left(\tilde{M}_{+}\right) \cong \frac{S O(n+2)}{S O(2) \times S O(n)} \cong Q_{n}(\mathbb{C}), \\
& M_{-}=\pi\left(\tilde{M}_{-}\right) \cong \frac{S O(n+2)}{S(O(1) \times O(n+1))} \cong \mathbb{R} P^{n+1} .
\end{aligned}
$$


Under a surjective linear map $(d \pi)_{p}: T_{p} \tilde{M}_{t} \rightarrow T_{p} M_{t}$, we have

$$
\begin{aligned}
& \operatorname{Ker}\left((d \pi)_{p}\right)=\mathbb{R} J \mathbf{x}(p) \subset \tilde{V}_{1}(p) \oplus \tilde{V}_{3}(p)=\mathbb{R} J \mathbf{x}(p) \oplus \mathbb{R} J \mathbf{n}(p), \\
& (d \pi)_{p}\left(\tilde{V}_{1}(p) \oplus \tilde{V}_{3}(p)\right)=V_{1}(p), \\
& (d \pi)_{p}\left(\tilde{V}_{2}(p)\right)=V_{2}(p), \quad(d \pi)_{p}\left(\tilde{V}_{4}(p)\right)=V_{3}(p) .
\end{aligned}
$$

The positive focal map $\nu_{+}: M_{t} \rightarrow M_{+}$is an $S O(n+2)$-equivariant submersion

$$
\nu_{+}: M_{t} \cong \frac{S O(n+2)}{\mathbb{Z}_{2} \times S O(n)} \longrightarrow M_{+}=Q_{n}(\mathbb{C}) \cong \frac{S O(n+2)}{S O(2) \times S O(n)} .
$$

Note that It is not a Riemannian submersion and the Reeb flow of a real hypersurface $M_{t}$ as an almost contact metric manifold is not isometric.

We observe that the differential of the positive focal map $\nu_{+}$has kernel $\operatorname{Ker}\left(d \nu_{+}\right)=V_{1}$. Let $\omega_{Q_{n}(\mathbb{C})}$ denote the Kähler form of $Q_{n}(\mathbb{C}) \subset \mathbb{C} P^{n+1}$. Then a relation between the pull-back form of $\omega_{Q_{n}(\mathbb{C})}$ by $\nu_{+}$and the restriction of the Kähler form $\omega$ of $\mathbb{C} P^{n+1}$ to $M_{t}$ is given as follows:

\section{Lemma 4.1.}

$$
\nu_{+}^{*} \omega_{Q_{n}(\mathbb{C})}=\left.\frac{1}{\cos \left(2 \theta_{1}\right)} \omega\right|_{M_{t}} .
$$

Here note that $\cos \left(2 \theta_{1}\right) \neq 0$.

The vector field $J(\operatorname{grad} f)$ on $\mathbb{C} P^{n+1}$ satisfies

$$
\omega(J(\operatorname{grad} f), \cdot)=g(\operatorname{grad} f, \cdot)=-d f,
$$

and hence it is a Hamiltonian vector field $X_{-f}$ on $\mathbb{C} P^{n+1}$ corresponding to the Hamiltonian $-f$. For each $t \in(-1,1)$, the flow of a Hamiltonian vector field $X_{-f}$ preserves the level set $f^{-1}(t)=M_{t}$ and $\left.X_{-f}\right|_{M_{t}}=J(\operatorname{grad} f)=\|\operatorname{grad} f\| J \nu$ belongs to $V_{1} \subset T M_{t}$. Note that $\|\operatorname{grad} f\|$ is constant on $M_{t}$. Hence we see that the vector field $\left.X_{-f}\right|_{M_{t}}$ generates the $S^{1}$-action on $M_{t}$ and the orbits of the $S^{1}$-action coincide with the fibers of the positive focal map $\nu_{+}: M_{t} \rightarrow M_{+}$. Therefore $M_{+}=Q_{n}(\mathbb{C})$ is regarded as a symplectic quotient $f^{-1}(t) / S^{1}$, but which is not a Kähler quotient, because the $S^{1}$-action on $M_{t}$ is not isometric.

$$
\begin{aligned}
& M_{t}\left(\cong \frac{S O(n+2)}{\mathbb{Z}_{2} \times S O(n)}\right)=f^{-1}(t) \subset \mathbb{C} P^{n+1} \\
& \nu_{+} \mid S^{1}\left(\cong S O(2) / \mathbb{Z}_{2}\right) \\
& M_{+}=Q_{n}(\mathbb{C})\left(\cong \frac{S O(n+2)}{S O(2) \times S O(n)}\right)=f^{-1}(t) / S^{1}
\end{aligned}
$$

Lemma 4.1 gives the relation between the push-forward symplectic form $\left(\nu_{+}\right)_{*} \omega$ on the symplectic quotient $f^{-1}(t) / S^{1}$ and the original Kähler form $\omega_{Q_{n}(\mathbb{C})}$ of $Q_{n}(\mathbb{C})$.

The positive focal map $\nu_{+}: M_{t} \rightarrow M_{+}=Q_{n}(\mathbb{C})$ can be considered as a principle fiber bundle with structure group $S^{1}$ over $Q_{n}(\mathbb{C})$. We define a 1 -form $\alpha_{\nu}$ on $M_{t}$ by

$$
\alpha_{\nu}(X):=\omega(\nu, X)=g(J \nu, X)
$$


for each $X \in T M_{t}$. The 1-form $\alpha_{\nu}$ satisfies

$$
\left(\nabla_{X} \alpha_{\nu}\right)(Y)=g\left(A_{\nu}(X), J Y\right)
$$

for each $X, Y \in T M_{t}$. Hence the exterior derivative of $\alpha_{\nu}$ is

$$
\begin{aligned}
\left(d \alpha_{\nu}\right)(X, Y) & =\left(\nabla_{X} \alpha_{\nu}\right)(Y)-\left(\nabla_{Y} \alpha_{\nu}\right)(X) \\
& =g\left(A_{\nu}(X), J Y\right)-g\left(A_{\nu}(Y), J X\right) \\
& =-\omega\left(A_{\nu}(X), Y\right)+\omega\left(A_{\nu}(Y), X\right)
\end{aligned}
$$

for each $X, Y \in T M_{t}$. Hence by using (4.1), (4.3), we have a formula

\section{Lemma 4.2.}

$$
d \alpha_{\nu}=-\left.2 \tan \left(2 \theta_{1}\right) \omega\right|_{M_{t}} .
$$

The principal $S^{1}$-bundle $\nu_{+}: M_{t} \rightarrow M_{+}=Q_{n}(\mathbb{C})$ has a connection invariant under the left group action of $S O(n+2)$ whose horizontal subspaces are defined by $(J \nu)^{\perp}=V_{2} \oplus V_{4}=V_{2} \oplus J\left(V_{2}\right)$. Then Lemma 4.2 means that its connection form is $\alpha_{\nu}$ and its connection form is $-\left.2 \tan \left(2 \theta_{1}\right) \omega\right|_{M_{t}}$. Moreover, by using Lemma 4.1 we have

$$
d \alpha_{\nu}=-2 \tan \left(2 \theta_{1}\right) \cos \left(2 \theta_{1}\right) \nu_{+}^{*} \omega_{Q_{n}(\mathbb{C})}=-2 \sin \left(2 \theta_{1}\right) \nu_{+}^{*} \omega_{Q_{n}(\mathbb{C})} .
$$

Note that $\sin \left(2 \theta_{1}\right) \neq 0$.

Let $\varphi: L \rightarrow Q_{n}(\mathbb{C})$ be a Lagrangian immersion of an $n$-dimensional smooth manifold $L^{n}$ into an $n$-dimensional complex hyperquadric $Q_{n}(\mathbb{C})$. Then we take a pull-back $S^{1}$-bundle $\hat{L}^{n+1}=\varphi^{-1} M_{t}$ by $\varphi$ over $L$ as

$$
\begin{aligned}
& \hat{L}^{n+1}=\varphi^{-1} M_{t} \stackrel{\hat{\varphi}_{t}}{\longrightarrow} M_{t}=f^{-1}(t) \quad \subset \mathbb{C} P^{n+1} \\
& \begin{array}{lll}
\nu_{+} \mid S^{1} & & \nu_{+} \mid S^{1} \\
L^{n} \stackrel{\varphi}{\longrightarrow} & M_{+}=Q_{n}(\mathbb{C}) \quad \subset \mathbb{C} P^{n+1}
\end{array}
\end{aligned}
$$

Since $\varphi$ is a Lagrangian immersion, i.e. $\varphi^{*} \omega_{Q_{n}(\mathbb{C})}=0$, by Lemma $4.1 \hat{\varphi}_{t}^{*} \omega=$ $\cos \left(2 \theta_{1}\right) \hat{\varphi}_{t}^{*} \nu_{+}^{*} \omega_{Q_{n}(\mathbb{C})}=\cos \left(2 \theta_{1}\right) \nu_{+}^{*} \varphi^{*} \omega_{Q_{n}(\mathbb{C})}=0$ and hence $\hat{\varphi}_{t}: L^{n} \rightarrow \mathbb{C} P^{n+1}$ is also a Lagrangian immersion. Moreover, $\nu_{+}: \hat{L}^{n+1}=\varphi^{-1} M_{t} \rightarrow L^{n}$ is also a principal $S^{1}$-bundle over $L^{n}$ and the pull-back connection is flat.

Theorem. If $\varphi: L \rightarrow Q_{n}(\mathbb{C})$ is a Lagrangian immersion into an $n$-dimensional complex hyperquadric, then we have a family of Lagrangian immersions $\hat{\varphi}_{t}$ : $\hat{L}=\varphi^{-1} M_{t} \rightarrow\left(M_{t} \subset\right) \mathbb{C} P^{n+1}(t \in(-1,1))$ into an $(n+1)$-dimensional complex projective space.

Remark 1 . The infinitesimal Lagrangian deformation given by the above family corresponds to the closed 1 -form $\hat{\varphi}_{t}^{*} \alpha_{\nu}$.

Remark 2. If we take the Gauss images of compact isoparametric hypersurfaces in $S^{n+1}(1)$ as $L^{n} \subset Q_{n}(\mathbb{C})$, then by this theorem we obtain a class of compact Lagrangian submanifolds $\nu_{+}^{*}\left(L^{n}\right)\left(\subset M_{t}\right)$ in $\mathbb{C} P^{n+1}$ related to isoparametric hypersurfaces. If we take compact homogeneous Lagrangian submanifolds as 
$L^{n} \subset Q_{n}(\mathbb{C})$, then by this theorem we obtain a class of compact Lagrangian orbits in $\mathbb{C} P^{n+1}$ which are not necessarily homogeneous in our sense.

We shall discuss the case when $L^{n}=S^{n} \subset Q_{n}(\mathbb{C})$ is a totally geodesic Lagrangian submanifold which is one of real forms in $Q_{n}(\mathbb{C})$. It also coincides with the Gauss image of a compact isoparametric hypersurface with $g=1$ in $S^{n+1}(1)$ (i.e. a great or small hypersphere of $S^{n+1}(1)$ ) in Section 4 .

$$
\begin{aligned}
& \tilde{L}^{n+1}=\left(\nu_{+}\right)^{-1}\left(S^{n}\right) \subset M_{t}=f^{-1}(t) \quad \subset \mathbb{C} P^{n+1} \\
& \nu_{+} S^{1} \quad \nu_{+} \downarrow S^{1} \\
& L^{n}=S^{n} \quad \subset \quad M_{+}=Q_{n}(\mathbb{C}) \quad \subset \quad \mathbb{C} P^{n+1}
\end{aligned}
$$

In this case by direct computations we obtain

Theorem . If $L^{n}=S^{n} \subset Q_{n}(\mathbb{C})$, then then we have a family of compact Lagrangian submanifolds $\hat{L}_{t}^{n+1}=\left(\nu_{+}\right)^{-1}\left(S^{n}\right)\left(\subset M_{t}\right)(t \in(-1,1))$ embedded in $\mathbb{C} P^{n+1}$ with the following properties:

(1) $\hat{L}_{t}^{n+1}$ is diffeomorphic but not isometric to the Riemannian product $S^{n} \times$ $S^{1}$ :

$$
\hat{L}_{t}^{n+1}=\nu_{+}^{-1}\left(S^{n}\right) \cong \frac{S O(n+1)}{S O(n)} \cdot\left(S O(2) \times\left\{\mathrm{I}_{n}\right\}\right) \cong S^{n} \times S^{1} .
$$

(2) $\hat{L}_{t}^{n+1}$ is a compact Lagrangian orbit of $S O(n+1) \times R^{1}$ but not homogeneous in the sense of Section 2. Here $R^{1}$ denotes the flow on $\mathbb{C} P^{n+1}$ generated by the vector field $J(\operatorname{grad} f)$.

(3) $\hat{L}_{t}^{n+1}$ is not Hamiltonian minimal.

Remark 3. $\hat{L}_{t}^{n+1}$ is isometric to a warped product $S^{1} \times_{\rho} S^{n}$ for a smooth function $\rho(\gamma)=\cos ^{2} \gamma \cos ^{2} \theta_{1}+\sin ^{2} \gamma \sin ^{2} \theta_{1}$ on $S^{1}$.

Finally we remark that the argument of this paper works also in the following other homogeneous isoparametric families:

(1) Construction from Lagrangian submanifold in $Q_{n}(\mathbb{C})$ to Lagrangian submanifolds in $Q_{n+1}(\mathbb{C})$ :

$$
\begin{array}{ccccc}
Q_{n+1}(\mathbb{C}) & = & Q_{n+1}(\mathbb{C})= & Q_{n+1}(\mathbb{C}) \\
\cup & \stackrel{\cup}{\cup} M_{-1}=S^{n+1} & \stackrel{\nu_{-}}{\cup} & M_{t} \stackrel{\nu_{+}}{\longrightarrow} & Q_{n}(\mathbb{C})=M_{1}
\end{array}
$$

(2) Construction from Lagrangian submanifold in $\mathbb{C} P^{n}$ to Lagrangian submanifolds in $\mathbb{C} P^{n+1}$ :

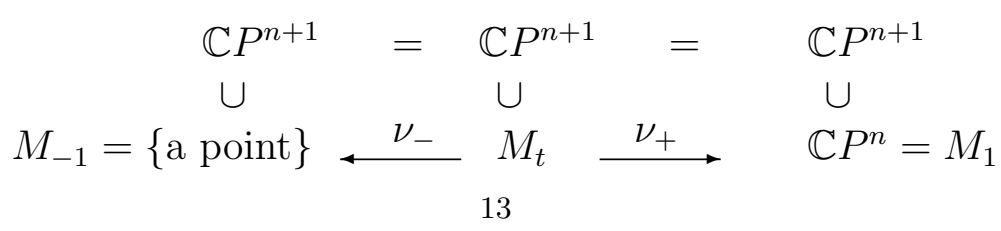




\section{REFERENCES}

[1] A. Amarzaya and Y. Ohnita, Hamiltonian stability of certain minimal Lagrangian submanifolds in complex projective spaces, Tohoku Math. J. 55 (2003), 583-610.

[2] A. Amarzaya and Y. Ohnita, Hamiltonian stability of parallel Lagrangian submanifolds embedded in complex space forms, a preprint, http://www.sci.osakacu.ac.jp/ ohnita/paper/Amar-Ohnita08.pdf.

[3] L. Bedulli and A. Gori, A Hamiltonian stable minimal Lagrangian submanifolds of projective spaces with nonparallel second fundamental form, Transf. Groups 12 (2007), 611-617.

[4] L. Bedulli and A. Gori, Homogeneous Lagrangian submanifolds, Comm. Anal. Geom. 16 (2008), 591-615.

[5] P. Dazord, Sur la geometrie des sous-fibres et des feuilletages lagrangiens, (French) [On the geometry of subbundles and Lagrange foliations] Ann. Sci. École Norm. Sup. (4) 14 (1981), no. 4, 465-480 (1982).

[6] Y.-X. Dong, Hamiltonian-minimal Lagrangian submanifolds in Kaehler manifolds with symmetries, Nonlinear Analysis 67 (2007), 865-882.

[7] H. Ma and Y. Ohnita, On Lagrangian submanifolds in complex hyperquadrics and isoparametric hypersurfaces in spheres. Math. Z. 261 (2009), 749-785.

[8] H. Ma and Y. Ohnita, Differential Geometry of Lagrangian Submanifolds and Hamiltonian Variational Problems. in Harmonic Maps and Differential Geometry, Contemporary Mathematics vol. 542, Amer. Math. Soc., Providence, RI, 2011, pp. 115-134.

[9] H. Ma and Y. Ohnita, Hamiltonian stability of the Gauss images of homogeneous isoparametric hypersurfaces, a preprint (2010), OCAMI Preprint Ser. no.10-23.

[10] H. F. Münzner, Isoparametrische Hyperfläche in Sphären, Math. Ann. 251 (1980), $57-71$.

[11] H. F. Münzner, Isoparametrische Hyperfläche in Sphären, II, Math. Ann. 256 (1981), 215-232.

[12] Y. G. Oh, Volume minimization of Lagrangian submanifolds under Hamiltonian deformations, Math. Z. 212 (1993), 175-192.

[13] Y. Ohnita, Stability and rigidity of special Lagrangian cones over certain minimal Legendrian orbits, Osaka J. Math. 44 (2007), 305-334.

[14] Y. Ohnita, Geometry of Lagrangian Submanifolds and Isoparametric Hypersurfaces, Proceedings of The Fourteenth International Workshop on Differential Geometry, 14 (2010), pp 43-67, NIMS, KMS and GRG. (OCAMI Preprint Ser. no.10-9.)

[15] Y. Ohnita, Certain Lagrangian submanifolds in Hermitian symmetric spaces and Hamiltonian stability problems, Proceedings of The Fifteenth International Workshop on Differential Geometry, 15 (2011), pp 209-234, ed. by Y.-J. Suh, NIMS, KMS and GRG. (OCAMI Preprint Ser. no.11-14).

[16] H. Ozeki and M. Takeuchi, On some types of isoparametric hypersurfaces in spheres I. Tohoku Math. J.(2) 27 (1975), 515-559.

[17] H. Ozeki and M. Takeuchi, On some types of isoparametric hypersurfaces in spheres II. Tohoku Math. J.(2) 28 (1976), 7-55.

[18] B. Palmer, Hamiltonian minimality of Hamiltonian stability of Gauss maps, Diff. Geom. and its Appl. 7 (1997), 51-58.

[19] D. Petrecca and F. Podesta, Construction of homogeneous Lagrangian submanifolds in $\boldsymbol{C P}^{n}$ and Hamiltonian stability, Tohoku Math. J.(2) 64 (2012), 261-268.

[20] R. Takagi, Real hypersurfaces in a complex projective space with constant principal curvatures. J. Math. Soc. Japan 27 no. 4 (1975), 43-53.

[21] R. Takagi, Real hypersurfaces in a complex projective space with constant principal curvatures II. J. Math. Soc. Japan 27 no. 4 (1975), 507-516. 
Department of Mathematics, Osaka City University, \& Osaka City University Advanced Mathematical Institute, Sugimoto, Sumiyoshi-Ku, Osaka, 5588585, JAPAN

E-mail address: ohnita@sci.osaka-cu.ac.jp 